\title{
Ant Colony Optimization (ACO) Based MANET Routing Protocols: A Comprehensive Review
}

\author{
Ahmad Khader Habboush ${ }^{1}$ \\ ${ }^{1}$ Jerash University, Jerash, Jordan \\ Correspondence: Ahmad Khader Habboush, Jerash University, Jerash, Jordan. E-mail: \\ ahmad_ram2001@yahoo.com
}

Received: October 24, 2018

Accepted: November 8, 2018

Online Published: January 26, 2019

doi:10.5539/cis. v12n1p82

URL: https://doi.org/10.5539/cis.v12n1p82

\begin{abstract}
Application of mobile ad hoc networks (MANETs) has gained significant popularity among researchers in the field of data communication networks. However, a MANET operating in a wireless environment imposes a number of challenges for the implementers so far as routing of packets across it is concerned. There is a wide range of research contributions are available in the literature wherein authors propose various solutions to overcome the problems and bottleneck related to routing in MANET. Especially soft computing techniques and Ant Colony Optimization (ACO) in particular has been significantly popular among the researchers to resolve MANET routing issues. This technique plays a vital role in route discovery in particular. In this paper, we have conducted a comprehensive review of this technique applied to routing in MANET with respect to various criteria. Hopefully this paper serves to a perfect document for researchers in this field.
\end{abstract}

Keywords: MANET, Routing, ACO, Ant Agents, FANT, BANT

\section{Introduction}

Mobile Ad Hoc Networks (MANETs) have found wide implementation in real world applications that present a series of challenges to successful implementation of it. The basic issue that evolves around implementation of MANETs is to design an optimal MANET routing protocol that overcomes various challenges pertaining to it. Varieties of approaches have been proposed by authors for designing MANET routing protocols[1,2,3]. However, Ant Colony Optimization ACO) based routing protocols appear to be more efficient in comparison with other approaches.

$\mathrm{ACO}$ as an Artificial Intelligence (AI) based search technique mostly relies on the past search results in order to find a path between the desired source and destination nodes in a MANET. Artificial ants being used for this purpose may lead to reinforcement that may consequently result in a premature convergence of the path finding procedure which can be overcome using randomization techniques in order for decision making during route discovery $[4,5]$. In ACO technique, a group of artificial ants work collaboratively in order to find an optimal solution to a given optimization problem by virtue of their communication that mimics the communication behaviour of real ants. An optimal path between source and destination can be achieved on the basis of concentration of pheromone deposited by the ants along the path of their traversal. ACO represents a heuristic approach that takes into consideration all the ants available in the network [6]. In comparison with optimization techniques like Genetic Algorithm (GA) and Simulated Annealing (SA), ACO exhibits better performance in finding a shortest path to the destination node [7]. Artificial ants used in ACO represent control packets that are used for collecting information while moving between source and destination nodes. Here, every node in the network stores a routing table that holds an entry for each of its neighbouring nodes. Each such entry refers to a pheromone value along with a set of variables and these values are used by the node in order to determine the goodness of a neighbouring node among all its neighbours. Out of multiple paths available between the source and destination nodes, the optimal one can be chosen with respect to the pheromone value.

\section{Classification of ACO Based MANET Routing Protocols}

ACO based MANET routing protocols are classified into the following categories with respect to their service goals.

Basic ACO Routing Protocols; 
- QoS Based ACO Routing Protocols;

- Power Based ACO Routing Protocols;

- Location Based ACO Routing Protocols;

- Security Based ACO Routing Protocols.

\subsection{Basic ACO Routing Protocols}

Various basic ACO based MANET routing protocols proposed by different authors are elaborated in this subsection as detailed below.

\subsubsection{Adaptive Agent Based ACO Routing}

Authors Caro G. D. and Dorigo M. Proposed Adaptive Agent based Routing Algorithm (AntNet) that is used for resolution of routing issues in MANET [8]. Here, authors use two categories of agents namely, Forward Ants (FANT) and Backward Ants (BANT), that are used for an optimal route selection between the communicating nodes using a proactive or table driven routing approach. FANTs are generated by the source node and transmitted to random destinations and on reaching the destination node, a FANT is dropped and in turn, a BANT is generated and transmitted back to the source along the path the respective FANT travelled. During travel back to the source, BANT updates the routing table entries at each node it traverses through. In this procedure, hop delay is chosen as the parameter that is updated in the pheromone table at each node.

\subsubsection{Ant Routing Algorithm}

Ant Routing Algorithm (ARA) belongs to the type of ACO based routing algorithms that uses a reactive approach to path discovery, has been proposed by authors in [9]. It comprises of three phases namely, route discovery, route maintenance and link failure handling. Like in AntNet, it makes use of agents FANT and BANT. A FANT is broadcast by the sender and it holds a unique identification number. Each intermediate node on receiving the FANT for the first time, creates an entry for it in the routing table with a triple comprising of three components: destination address, next hop and pheromone value $(\Omega)$. Duplicate FANTs with the same sequence numbers are ignored by the intermediate node. The pheromone value at a node is calculated on the basis of number hops that the FANT has travelled before reaching the respective node. When a FANT reaches the destination node, it is converted to a BANT and sent back to the source and when source receives the BANT, a path is established between the source and destination nodes. When a node vi sends a packet to destination node vd via intermediate node $\mathrm{vj}$, the entry $(\mathrm{vd}, \mathrm{vj}, \Omega)$ in the pheromone table is updated as $(\mathrm{vd}, \mathrm{vj}, \Delta \Omega)$. Similar update is made when BANT travels back to source node vs. A route failure is inferred following missing acknowledgements at MAC layer of source node. In such a case, an alternate path is searched. If no alternate paths are available at that point of time, a route discovery procedure is initiated as described earlier.

\subsubsection{Probabilistic Emergent Routing Algorithm}

A proactive routing algorithm called Probabilistic Emergent Routing Algorithm (PERA) for route discovery and maintenance in MANET was proposed by authors in [19]. It also implements ant like agents for the purpose of route discovery and maintenance. A node in this strategy initially as well as periodically with a fixed interval of time, uses HELLO messages for discovering its neighbours at single hop distance from it. In the beginning, each entry in the routing table of a node is initialized with a probabilistic value that is exactly the inverse of its number of one hop neighbours. This protocol makes use of three types of ant agents namely, regular FANTs, Uniform FANTs (UFANTs) and BANTs. Both Regular FANTs and UFANTs are generated by the source node for route discovery in the same way as described in AntNet and ARA using proactive approach. However, UFANTs are especially used for discovery of a new route after an already discovered one is discarded for some or other reasons. After a route is established, data transmission takes place with reference to the probabilistic values stored with each intermediate node.

\subsubsection{Ant-Based Hybrid Routing Algorithm}

Ant-Based Hybrid Routing Algorithm for MANET (AntHocNet) was proposed by authors in [11]. It represents a hybrid multipath routing algorithm that uses six different agents namely, Proactive FANT (PFANT, Reactive FANT (RFANT), Proactive BANT (PBANT), Reactive BANT (RBANT), Repair FANT (ReFANT) and Repair BANT (ReBANT). A source node may unicast or broadcast RFANTs in the beginning of path setup procedure that implements a reactive approach. An intermediate node after receiving several FANTs, chooses the best one on the basis of the number of hops the FANt has travelled and its travel duration. Selected thus FANTs are sent to the destination node. This procedure helps in selecting the optimal path. As in AntNet or ARA, after RFANT is received by the destination node, it generates RBANT that is sent back to the source node along the path that the 
respective RFANT traversed before reaching the destination. During its travel, RBANT updates the pheromone table entries at the intermediate nodes. When RBANT reaches the source node, an optimal path is established. Following this procedure, several optimal paths can be established between the source and destination nodes. This protocol relies on a stochastic data routing strategy for datra transmission by virtue of which good and optimal paths can be selected automatically.

\subsubsection{ImProved Ant Colony Optimization Routing Algorithm for Mobile Ad Hoc Networks}

ACO based routing named imProved Ant Colony Optimization routing algorithm for mobile ad hoc NETworks (PACONET) has been proposed by authors in [12]. Here, a few restrictions are imposed on Reactive FANTs as detailed below. Here, the size of the routing table stored at each node equals to the product of node degree and the number of nodes in the network. In this table, a row is assigned for each neighbour of the node and a column for each other node in the network that is not a neighbour of it. Each entry holds a pair of values: a binary value that refers to status node visited and the other as pheromone concentration value. During the process of route discovery, a FANT selects the next node with reference to the routing table with highest pheromone concentration value and the next hop from node unvisited status thereby avoiding cycles if any. FANt records the travel time along with the list of nodes visited in its memory thereby updating the routing pheromone value at each intermediate node. When FANt reaches the desired destination, it is dropped and a BANT is generated that is sent back to the source node making use of the information collected by the respective FANT. FANT on its way to source node updates the pheromone value at each intermediate node too. When FANT reaches the source node, the route is established.

\subsubsection{ACO Based Hybrid Routing Algorithm}

W. Yu has proposed a routing algorithm named Ant Colony Optimization based Hybrid Routing Algorithm (ACOHRA) that relies on reactive strategy for route setup and proactive strategy for route maintenance in MANET. In this approach, each node stores a routing table each entry of which holds a probabilistic value called pheromone. It makes use of two categories of agents: ant agents for collecting information and service agents for updating pheromone value. During route discovery, source generates FANTs that uses reactive procedure while travelling to destination thereby recording the list of nodes of its traversal. At each intermediate node, FANT uses the information available in the routing table in order to choose the next hop. After FANT reaches the destination, it is replaced by a BANT that is sent back to the source as per the list of nodes recorded by the FANT during its traversal to destination. At the same time, BANT also calculates the delay from each node to the destination. After BANT reaches the source node, it is killed and the route is setup. The service agents here are used for updating the pheromone values at intermediate nodes with reference to the information collected by ant agents. In addition, proactive ant agents are used here for route maintenance. Further, AODV mechanism is used for handling link failures and their repair.

\subsubsection{Hybrid ant colony Optimization algorithm for mobile ad hoc NETworks}

A zone based ACO routing protocol for MANETs called Hybrid and colony optimization algorithm for mobile ad hoc NETworks (HOPNET) has been proposed by authors in [14]. In this approach, a network is partitioned into different zones. Within a zone, path setup is achieved using proactive routing whereas reactive routing strategy is used for path setup between separate zones. A zone here is defined with respect to the local neighbourhood of a node within a predefined radius that is expressed in terms of number of hops. The neighbouring nodes are split into three types depending on their position as, internal, external and boundary nodes. The basic mechanism of this protocol is in fact a combination of protocols AntNet discussed earlier and Zone Routing Protocol (ZRP). Each node in the network maintains two separate routing tables. Intrazone routing table (IntraRT) is maintained proactively in order to store the information regarding topology of the nodes within the zone. It holds the routes to external nodes. Ant agents like internal FANTs (IFANTs) and internal BANTs (IBANTs) facilitate in establishing a route within the zone itself using proactive strategy thereby updating the Intrazone routing table. To find a route to a node that exists beyond the zone, external FANTs (EFANTs) and external BANTs (EBANTs) are used in the same manner making use of a reactive approach. When the current route breaks, alternate paths can be chosen during which the data packets are buffered until the alternate route is established. In the process of finding an alternate route, a notification Ant (NANT) is sent to the source in order to inform the source regarding the new route so that it can send the packets along this route.

\subsubsection{Ant Routing Protocol for MANET}

Ant routing protocol for MANET (ANT-E) that makes use of a search procedure called Blocking Expanding Ring Search (Blocking-ERS) has been proposed by authors in $[15,16]$. Blocking -ERS mechanism is used in this protocol in order to overcome the overhead associated with route discovery process that involves route request messages passing via all intermediate nodes during this process. It implements negative acknowledgement (NACK) 
to achieve better performance in routing of data packets. Here, a routing table holding the information such as destination address, next hop and pheromone value, is stored with each node in the network. In this protocol, when route discovery is initiated, source sends a FANT to all its neighbours at one hop distance and the process is repeated subsequently until the desired destination is reached. During this process, each intermediate node maintains a copy of the route request packet so that duplicates can be discarded in future. This reduces the overhead significantly unlikely in other approaches. On receiving the FANT, the destination node drops it, in turn creates a BANT sends it back to the source. When BANT reaches the source, the route discovery process terminates and the route is established.

\subsection{QoS Aware Routing Protocols}

In this subsection, we discuss several QoS aware ACO routing protocols that are detailed below.

\subsubsection{Probability Routing Algorithm for Mobile Ad-hoc Networks Resource Management}

A QOS aware MANET routing algorithm called "Ant Routing Algorithm for Mobile Ad-hoc networks (ARAMA)" has been proposed by authors in [17]. It uses a proactive approach for routing on the basis of information collected by the ants regarding the path. Source generates FANTs that depend upon the network characteristics such as delay, data rate etc. And sent to the destination using proactive routing strategy with reference to the probability routing table stored at each node in the network. During traversal, FANT records local as well as global path information regarding the QoS parameters like delay, bandwidth, hop count and remaining battery life. When FANT reaches the destination node, it is killed and in turn, a BANT is generated. The global information held by the FANT are analyzed and a normalized link index is computed that characterizes if the path is good or bad to be selected for data transfer. BANT carrying thus computed link index travels back to the source thereby updating the pheromone values at each intermediate node along its path of traversal. After BANT is received at source node, the routing table of the source node is updated accordingly and as a result, multiple paths to the destination are stored at the source node. This algorithm implements two categories of ant agents named as "Negative Backward Ants (NBAs)" and "Destination Trail Ants (DTAs)". These two ant agents are used for improving the performance of this algorithm. When FANT while travelling to the destination encounters a loop or the Time-To-Live (TTL) for the packet expires, FANT is killed and a NBA is generated and sent back to the source that indicates to a bad link index for the path. This algorithm is capable of reducing the time required for connection setup and at the same time, delivery is ensured here.

\subsubsection{Dynamic Ant Colony Based Routing Algorithm}

A DSR based routing protocol called "Dynamic Ant Colony Based Routing Algorithm (SAMP-DSR)" for routing in MANET is proposed by the authors in [18]. It comprises of two principal components: first component is a proactive one that uses ACO technique and the second one is a reactive one used for path discovery. The first component is used for finding an optimal route to destination that seamlessly runs in the background. How good ia a path can be assessed in the context of end-to-end reliability that is computed as a function of the trip time of the ants. The second component participates during the route request. In the process of route discovery, the source node takes measure of a list of parameters such as number of paths n0, guaranteed minimum reliability $\mathrm{Pu}$, time window for end-to-end reliability tw and based on these parameters calculates the reliability of the path at each node till the destination node is reached. At the destination, the paths with minimum path reliability (Plower) are arranged into a list called CandidateSet in decreasing order. Then the list CandidateSet is reduced to TraceSet that holds only the disjoint paths. This process continues until a desired number of paths are found between the communicating nodes. This algorithm functions in two modes namely, local mode and ACO mode, where the algorithm may switch between these two modes during path discovery. In local mode, the local information are collected by nodes from their neighbours and in ACO mode, the global information along with the route preferences are gathered thereby reducing routing overhead to minimum.

\subsubsection{QoS Enabled Ant Colony Based Multipath Routing Protocol}

A routing protocol that relies on selection of next hop availability along with probability of path preference called "QoS enabled Ant colony based Multipath Routing (QAMR)" has been devised by authors in [10]. Here, an optimal path between source and destination is chosen on the basis of the imposed QoS constraints defined on the basis of parameters like number of hops, remaining network bandwidth and end-to-end delay. Next hop availability here is determined with respect to link availability taking into consideration node mobility and remaining battery power. This protocol guarantees better QoS although this algorithm is constrained by a number of control messages exchanged during path discovery. 


\subsubsection{QoS Routing Based on Ant Colony Optimization}

A routing protocol for multi-rate MANET called "QoS Routing based on Ant colony optimization (QoRA) was proposed by authors in [20]. This protocol makes use of Simple Network Management Protocol (SNMP) in order for measurement of QoS metrics [21]. This protocol mostly aims at reducing overhead resulting from information collection and congestion avoidance too. It uses two entities: QoRA entity for route setup as per the desired QoS and SNMP entity that is mainly used for information collection relating to the QoS parameters such as delay, bandwidth and packet loss ratio. QoRA entity generates three types of ant agents such as FANT, BANT and error ants (EANTs). It also brings updates in routing tables of neighbouring nodes with the QoS parameters like bandwidth (B), delay (D) and success rate (R). These updates are carried out with the help of SNMP ant agents. Here, SNMP agent collects the information regarding QoS parameters and updates making use of a "Management Information Base (MIB)". This algorithm operates in five different phases namely, forward phase, backward phase, packet forwarding phase, maintenance phase and link failure phase. These phases explicitly reveal the functionality of this protocol. As in many other routing algorithms detailed earlier, a source generates a FANT and sends it towards destination. At every node along the path to destination, only the best ant is forwarded to the next hop with respect to its goodness value. Destination on receiving the FANT, coverts it to a BANT and sends back to source along the same path that the respective FANT travelled. On the way to source, BANT collects information about link quality and updates the routing tables of nodes along the path. When BANT reaches the source, path is established following which data packets can be sent by source stochastically. Route maintenance is conducted by the SNMP agent.

\subsection{Location Based Routing Protocols}

In this subsection, we discuss several location based ACO based MANET routing protocols as elaborated below.

\subsubsection{Position Based Ant Colony Routing for MANET}

A location based ACO routing protocol for MANET called "POSition based ANT colony routing for mobile adhoc networks (POSANT)" is proposed by authors in [22]. It relies on a reactive routing procedure and operates on the basis of the position of nodes in the MANET. It focuses mainly on reducing the time needed for route setup and control message overhead associated with the process of route establishment. Here, every node in the network is aware of the positions of itself, its neighbours as well as the destination prior to initiating the process of route establishment. Here, the neighbouring nodes are split into three separate zones on the basis of the location information available with the communicating node. This separation of zones implements a greedy approach that enables selection of the shortest path to the destination node. In the process of route setup, FANTs are generated by source and sent reactively towards the destination node. Two separate tables namely, a pheromone table and back routing (BR) table, are stored with each node in the network. Every node after receiving the FANT, creates an entry in BR table with a sequence number, ID of the immediate sender of the FANT and destination address. An intermediate node decides stochastically to choose the next hop with reference to the pheromone table available with it. BANT is generated by destination node on receiving the FANT and the BANT travels back towards the source with the same sequence number as the respective FANT as per the information stored in BR table. After BANT is received at source, route is established. It should be noted here that in POSANT, each node operates in two modes namely, regular mode and broken mode. Initially, each node in the network assumes regular mode. When a link failure occurs, every node associated with the link switches to broken mode thereby informing to all its neighbours regarding this. The same process is repeated by every neighbour and subsequently, the information regarding link failure reaches every node that participates in the route.

\subsubsection{ACO Routing Based on Robustness of Ad Hoc Networks with GPSs}

ACO based routing approach for MANET with Global Positioning Systems (GPSs) has been proposed named as Robustness-ACO that operates on robustness of the network rather than any location information [23, 24]. In this approach, the information required for route establishment and its maintenance are collected from the visited nodes with the help of GPS. It operates on two types of ant agents namely, hybrid FANTs and hybrid BANTs. A hybrid FANT travels from source to destination like in the approaches detailed earlier thereby collecting path information and updating routing tables as well as pheromone tables of nodes visited along the path. Hybrid BANTs travelling backward update routing as well as pheromone tables of visited nodes thereby finding multiple paths between source and destination nodes. Every nodes stores a neighbour table in order for storing GPS information needed for predicting link failures and a routing table that holds pheromone values. The robustness of an entire path is computed from the minimum robustness recorded for all links between each pair of nodes. The robustness is calculated from the relative velocities and relative positions of nodes with reference to the pheromone values deposited by ant agents along the path. In case of a link failure especially in highly dynamic networks, GPS 
information are used for setup of alternate paths with a high probability and this makes this protocol significantly adaptive.

\subsubsection{Mobility Aware ACO Routing for MANET}

An adaptive routing for MANET called "Mobility aware Ant Colony Optimization Routing DYMO (MAR-DYMO) is proposed in [25]. It makes use of Nakagami Fading Model (NFM) in order for depositing pheromone [26]. Link stability in this protocol is determined using Kinetic Graph framework [27]. NFM is used here in order to calculate the probability that a message can be received within a specified range which can be achieved using Nakagami distribution along with a natural number called fading parameter. Pheromone value is computed with respect to the ratio of this probability value to the power threshold of the respective link. Here, HELLO messages are propagated non-periodically thereby avoiding useless broadcast of such messages and this process is controlled by Kinetic Graph frameworks. Selection of the next hop out of a number of neighbouring nodes is achieved with respect to the pheromone values stored at nodes using roulette-wheel selection method [28]. Each route in this approach can remain active only for a specific period of time and declared as invalid after the time period expires.

\subsubsection{Mobility Aware Zone Based ACO Routing for VANET}

A routing protocol for Vehicular Ad Hoc Networks (VANET) that is oriented around partition of the network into multiple zones, called as Mobility Aware Zone based Ant Colony Optimization Routing for VANET (MAZACORNET), has been devised by the authors in [29]. In this protocol, route establishment within a zone is carried out proactively whereas in order to communicate with nodes belonging to another zone, a path is found reactively. The pheromone model presented in this approach is exactly the same as in MAR-DYMO, but the difference lies in types of routing tables and ant agents implemented. Nodes used here are of three different categories: interior nodes that lie within a distance equal to less than the radius of the zone that the communicating node belongs to, boundary nodes that exactly exist on the boundary between two neighbouring zones and exterior nodes that exist in another zone. Five different types of ants are used here namely, internal forward ants IFANTs), external forward ants (EFANTs), Backward ants (BANTs), notification ants (NANTs) and error ants (EANTs). IFANTs update pheromone table during intra-zone routing. EFANTs and BANTs participate in discovering routes to the boundary nodes. Maintenance of routes within a zone is conducted using proactive approach, but in case of failure of an inter zone route, an alternate route must be established and subsequently must be informed to other nodes which subsequently update their inter zone routing table entries accordingly and these updates are carried out making use of NANTs. In case, an alternate route cannot be found, an error message is propagated to the source node using EANT. Pheromone value in this approach represents the sum of the value of link stability (LS) and the probability that the message is received successfully. Link stability is computed from the positions and velocities of the nodes participating in the link. Probability of successful receipt of message is calculated from the distance between the same ranges that is further calculated using NFM.

\subsubsection{Secure and Robust Multi-constrained QoS Aware Routing Algorithm}

In order for compliance of QoS and addressing the security issues pertaining to VANET, a novel routing protocol called "Secure and Robust Multi-constrained QoS Aware Routing Algorithm (S-AMCQ) has been proposed by authors in [30]. Here, in order to find route that comply with a specified QoS, ACO algorithm is used along with Extended-VANET oriented Evaluation Graph (E-VoEG) that is used for authentication purpose in order to guarantee defence against internal as well as external attackers respectively [31]. Ant agents used in this approach are of three types such as request ant (RQANT), route reply ant (RPANT) and route error ant (REANT). RQANTs and RPANTs participate in route discovery procedure. REANTs are used to send notifications to all the nodes in case of a link breakage. Route pheromone table is maintained at each node that facilitates routing of data as well as control packets. Data Signature (DS) mechanism used in this approach protects the control ants from external attackers in order to maintain their integrity as well as authenticity. During the route discovery process, first of all, source sends the Message Digest (MD) of RQANT that is obtained as a one way hash function, and then, it is attached to a Digital Signature (DS) which is generated making use of a pseudonymous certificate obtained from the Certification Authority (CA) along with a secret signing key of the node. At every intermediate node, the DS is verified and if it is found to be authentic, then RQANT is accepted by the node. This process is repeated at each intermediate node before it reaches the destination. When a RQANT that complies with the specified QoS, is received at the destination, a RPANT is generated and sent back to source along the same route that the RQANT traversed. When RPANT is received by an intermediate node, signature is verified, link quality of the path is assessed and the pheromone table is updated by the intermediate node. E-VoEG model is responsible for route maintenance and handling link breakages. When a REANT reports a link failure, its authenticity is verified with reference to the E-VoEG model. Then accordingly, either another route discovery is initiated of the transmission 
is switched to another valid route that complies with the specified QoS requirements.

\subsection{Power Aware Routing ACO Protocols}

In this subsection various power aware ACO based MANET routing protocols are described as proposed by different authors.

\subsubsection{Ant-Swarm Inspired Energy Efficient Ad-hoc On Demand Routing}

ACO based MANET routing protocol called "Ant-Swarm Energy Efficient Ad-hoc On Demand Routing (ACOEEAODR)" has been proposed by authors in [32]. This protocol assumes that every node in the network must possess the minimum required battery power level in order for its sustainability in the network. In this approach, an optimal path is chosen on the basis of remaining battery power available at a node and the number of hops to the destination. Here, the pheromone value at each node is updated with reference to the available battery power of the node. Although the two earlier specified parameters are considered for choosing an optimal path, more emphasis is given on the available battery power though. Next hop is chosen stochastically since it is known. Since this protocol is a reactive one route request (RREQ) and route reply (RREP) messages are used for route discovery as well as route maintenance.

\subsubsection{Energy Aware Ant Based Routing}

A MANET routing protocol called "Energy Aware Ant based Routing EAAR)" that uses AntHocNet protocol discussed earlier along with energy efficiency has been proposed by authors in [33]. This protocol aims at extending lifetime of a node in a MANET in the context of battery life in a multi-path scenario. Three tables are used in this approach namely, one neighbour table that stores the list of neighbouring nodes, one seen table that holds the information regarding the packets received by the node and a routing table containing the routing pheromone values. Route discovery is conducted in the same way as in case of AntHocNet elaborated earlier in this section. Sender broadcasts FANTs reactively. When FANT reaches the destination, it waits for the other FANTs to reach with the same delay as the first FANT. FANTs are killed and in turn, BANTs are generated and sent back to the source along the respective paths. Pheromone values are updated with respect to Maximum minimum Remaining Battery power (MRB) and the link hop count too. When source receives the first BANT, it waits for other BANTs to arrive with the same delay as the first one. When all BANTs arrive, they are killed and multiple paths thus are established following which data transmission can resume. Route maintenance is carried out using pheromone reinforcement and evaporation. In case of link failures, new route discovery is initiated.

\subsubsection{Robust Energy Efficient ACO Routing Algorithm for Multi-hop Ad-hoc Networks}

A routing protocol for multi-hop ad-hoc networks named "Robust Energy Efficient Ant Colony Optimization Algorithm for Multi-hop Ad-hoc Networks (AntHocMMP)" has been proposed by authors in [34]. It uses a MaxMin-Path (MMP) algorithm for establishment of optimal paths with the help of ant agents [35]. It operates in two separate phases namely, selection phase and energy conservation phase. During selection phase, multiple paths are setup with the help of MMP algorithm. MMP algorithm here uses energy level along with energy path cost in order for selection of a path. Zero energy level is termed as saturation energy level and a threshold energy level refers to a minimum energy level required for a node to remain alive in the network. Energy path cost between the source and destination nodes is computed from two separate cost functions namely, transmission cost and reception cost. Transmission cost is the total amount of energy consumed during travel between the communicating nodes. Reception cost is the amount of energy consumed with respect to Euclidian distance. During energy conservation phase, FANTs are sent via multiple paths from source to destination reactively. BANTs generated by destination travel back to source updating the pheromone values at nodes along the paths traversed. When BANTs are received at source, an optimal path is established between source and destination. This phase ensures energy level of nodes higher than the specified threshold that enhances the lifetime of nodes in the network.

\subsubsection{Ant Colony Based Energy Control Routing}

A protocol for MANET called "Ant Colony based Energy Control Routing (ACECR)" that relies on computation of residual battery power of nodes in order for route selection with the consideration of required average and minimum energy level for the route [36]. In the process of route discovery, FANTs are sent from source to destination and BANTs are sent from destination on receipt of FANTs. BANTs follow the same path as the respective FANTs travelling to source thereby updating pheromone values at nodes along the path on the basis of minimum energy at nodes, average energy of the path and the hop count of the path. When BANT reaches the source, route is established. 


\subsubsection{Hybrid ACO Routing}

A secure as well as energy aware MANET routing protocol that implements node trust values using Hybrid ACO has been proposed by authors in [37]. This approach aims at ensuring energy efficiency by virtue of even distribution of load among the nodes in the network thereby providing secure routing in MANETs. It relies on the optimization technique Simulated Annealing (SA) in order for selection of an optimal path between two nodes in the network. The pheromone value assigned to each node represents its trust value that ensures secure communication. Here, a FANT generated by source chooses the next hop on the basis of comparison of trust values of twenty five nodes at two-hop distance from it i.e., five of its own neighbours and five neighbours each from its own neighbours. This process implements SA procedure. Out of these twenty five nodes, an optimal path is determined that comprises of one node of one-hop distance and another of two-hop distance. Then, FANT moves to next node and updates the pheromone values. This process is repeated until the FANT reaches the destination and thus, an optimal shortest path can be determined.

\subsubsection{Energy Aware Multipath Routing Algorithm ACO-FDRPSO}

An energy aware multipath routing algorithm for MANET called ACO-FDRPSO has been proposed by authors in [38]. This protocol results from a combination of Hybrid-ACO detailed earlier and Fitness Distance Ratio based Particle swarm Optimization (FDRPSO) [39]. ACO is implemented here for extending the lifetime of nodes with the help of Duty Cycle algorithm and FDRPSO is used for minimization of energy consumed by the nodes in the network. Duty Cycle algorithm is capable of minimizing the rate of drainage of battery power of nodes that leads to extension of lifetime of nodes. During route discovery, ant agents select nodes that possess higher level of available residual energy as determined by Duty Cycle algorithm. Ant agents travel from source to destination and travel back to source thereby establishing multiple paths and during travel, these ant agents collect path information and update pheromone values at nodes visited. After multiple paths are discovered, an optimal one can be chosen to go ahead with data transmission subsequently.

\subsection{Security Based ACO Routing Protocols}

In this subsection, security based ACO routing protocols for MANET proposed by various authors are elaborated as follows.

\subsubsection{Secure AntNet Routing Algorithm for Scalable Ad Hoc Networks Using Elliptic Curve Cryptography}

A routing protocol for scalable MANETs called "Secure AntNet Routing using Elliptic Curve Cryptography (SARECC)" has been proposed by authors in [40]. This protocol uses ACO technique along with Elliptic Curve Cryptography (ECC) [41]. Here, every node makes an assessment of trust values of its neighbouring nodes where the trust value is directly proportional to the probability of packet delivery ratio for the node. AntNet algorithm is implemented by the source node in order to discover multiple paths to the destination. ECC is used here for a mutual authentication between the source and destination nodes. Trust value measurement here carried out on the basis of uncertainty rather than any pheromone value as elaborated in many of the earlier discussed routing algorithms.

\subsubsection{Secure Power Aware Ant Routing Algorithm}

A MANET routing protocol named "Secure Power Aware Ant Routing Algorithm (SPA-ARA)" has been proposed and detailed in [42]. In the beginning of route discovery process, source node sends FANTs towards destination. Here, FANT is attached with Message Authentication Code (MAC) that is generated by virtue of using HMAC keyed hash along with a shared group key [43]. At every intermediate node, validity of MAC is assessed and if it found to be valid, trust value of the sender of it is checked with reference to a predefined threshold value if it holds valid. Then, using a two-party key establishment protocol, a secret key is generated for the pair of communicating nodes. FANT is dropped otherwise. On successful validation of the above, FANT is sent to destination followed by a BANT being sent back to source. At every intermediate node, MAC attached with BANT is verified with the help of the secret key generated by the respective FANT earlier. In addition, BANT carries out updates to pheromone values at these nodes during travel to source. When BANT reaches source node, all the nodes along the path must have been authenticated and the path is established for data transmission further.

\subsubsection{ACO Based Prevention of Black-hole Attack in MANET Routing}

ACO based technique to achieve secure routing in MANET that uses ACO mechanism for prevention of blackhole attacks is proposed by authors in [44]. During route discovery, source node broadcasts forward agent (FA) that is attached with a digital signature (DS) of the source node. The neighbouring node after receiving FA, verifies the DS and on finding it to be valid broadcasts further. Through sequence of broadcasts, FA reaches destination thereby collecting path information during travel. At destination, FA is dropped and in turn, BA is generated being 
attached with DS of destination node and sent back to source along the path that FA traversed. Like FA, DS attached with BA is verified at every intermediate node and if found valid, forwarded to the next hop. When BA successfully reaches source node, the path is established. Performance of this approach is verified by the authors via simulation taking into consideration QoS parameters like packet delivery ratio (PDR) and packet loss (PL).

\subsubsection{Swarm Based Detection and Defence Techniques}

A MANET protocol for multipath routing that relies on swarm intelligence aspects of ACO technique for establishment of multiple paths named as "Swarm based intrusion Detection and Defence Technique (SBDT)" has been innovated by authors in [45]. Each node in the network is associated with a trust value and a node with higher trust value, residual energy as well as bandwidth is authorized to monitor its neighbouring nodes and considered as active node (NA). In course of data transmission, if the trust value of any of the neighbouring nodes is observed by NA to be lower than the threshold value fixed earlier, then NA declares that node as malicious thereby informing the source regarding this. However, updates of trust values in this approach are not specified by the authors here.

\section{Conclusion and Future Work}

In this paper, we have made an attempt to summarize the research contributions available for addressing the problem of routing in MANET. The main focus is given in this work at the research contributions of authors that use ACO technique in order for resolution of routing issues in MANET and in particular the route discovery process at the beginning of communication between two nodes in the network. Different criteria such as battery power, QoS, security aspects lye at the foundation s of proposed solutions by various authors. As it can be observed that in almost all proposals, FANTs and BANTs are used for route discovery and the process followed is the same across all proposed protocols with a few variations across them. Nevertheless, all solution converge to a perception that ACO technique could be one of the most efficient mechanism for resolving issues related to route discovery during communications between two nodes in MANET. This investigation can be further carried out in future to address several other techniques like evolutionary computing techniques pertaining to resolution of routing issues in MANET.

\section{References}

Al-Ani, A. D., \& Seitz, J. (2016). QoS-aware routing in multi-rate ad hoc networks based on ant colony optimization. Netw. Protocols Algorithms, 7(4), 1-25.

Back, T. (1996). Evolutionary Algorithms in Theory and Practice: Evolution Strategies, Evolutionary Programming, Genetic Algorithms. Oxford, U.K.: Oxford Univ. Press.

Baras, J. S., \& Mehta, H. (2003). A probabilistic emergent routing algorithm for mobile ad hoc networks. in Proc. Modeling Optim. Mobile, Ad Hoc Wireless Netw. (WiOpt), p. 10.

Caro, G. Di., \& Dorigo, M. (1998). AntNet: Distributed stigmergetic control for communications networks. J. Artif. Intell. Res., 9, 317-365.

Caro, G., Di, Ducatelle, F., \& Gambardella, L. M. (2005). AntHocNet: An adaptive nature-inspired algorithm for routing in mobile ad hoc networks, Eur. Trans. Telecommun., 16(5), 443-455.

Case, J. D., Fedor, M., Schoffstall, M. L., \& Davin, J. (1990). Simple network management protocol (SNMP). IETF, Fremont, CA, USA, Tech. Rep. rfc1157. Retrieved from http://www.rfc- editor.org/info/rfc1157

Correia, S. L. O. B., Celestino, J., \& Cherkaoui, O. (2011). Mobility-aware ant colony optimization routing for vehicular ad hoc networks. in Proc. IEEE Wireless Commun. Netw. Conf., Mar., pp. 1125-1130.

Dressler, F., \& Akan, O. B. (2010). A Survey on bio-inspired networking. International Journal of Computer Networks, 54(6), 881-900.

Eiza, M. H., \& Ni, Q. (2013). An Evolving Graph-Based Reliable Routing Scheme for VANETs. IEEE Trans. Vehicular Technology, 62(4), 1493-1504.

Eiza, M. H., Owens, T., \& Ni, Q. (2016). Secure and robust multi-constrained QoS aware routing algorithm for VANETs. IEEE Trans. Depend. Sec. Comput., 13(1), 32-45.

Gunes, M., Sorges, U., \& Bouazizi, I. (2002). ARA-the ant-colony based routing algorithm for MANETs, “ in Proc. Int. Conf. Parallel Process. Workshops, pp. 79-85.

Härri, J., Bonnet, C., \& Filali, F. (2008). Kinetic mobility management applied to vehicular ad hoc network protocols. Comput. Commun., 31(12), 2907-2924.

Hussein, O., \& Saadawi, T. (2003). Ant routing algorithm for mobile ad-hoc networks (ARAMA). in Proc. IEEE 
Int. Perform., Comput., Commun. Conf., pp. 281-290, Apr.

Indirani, G., \& Selvakumar, K. (2012). Swarm based detection and defense technique for malicious attacks in mobile ad hoc networks. Int. J. Comput. Appl., 50(19), 1-6.

Jagadev, A. K., Pattanayak, B. K., Mishra, M. K., \& Nayak, M. (2010). Power and Delay Aware On-demand Routing for Ad Hoc Networks. International Journal of Computer Science and Engineering (IJCSE), 2(4), 917-923.

Kadono, D., Izumi, T., Ooshita, F., Kakugawa, H., \& Masuzawa, T. (2010). An ant colony optimization routing based on robustness for ad hoc networks with GPSs. Ad Hoc Netw., 8(1), 63-76.

Kamali, S., \& Opatrny, J. (2007). POSANT: A position based ant colony routing algorithm for mobile ad-hoc networks. in Proc. 3rd Int. Conf. Wireless Mobile Commun. (ICWMC), p.21.

Kapoor, V., Abraham, V. S., \& Singh, R. (2008). Elliptic curve cryptography. Ubiquity, 5, 7:1-7:8.

Kathirvel, A. (2011). Introduction to GloMoSim. Saarbrücken, Germany: LAP Lambert Academic Publishing.

Khosrowshahi-Asl, E., Noorhosseini, M., \& Pirouz, A. S. (2011). A dynamic ant colony based routing algorithm for mobile ad-hoc networks. J. Inf. Sci. Eng., 27(5), 1581-1596.

Killat, M., \& Hartenstein, H. (2009). An empirical model for probability of packet reception in vehicular ad hoc networks. EURASIP J. Wireless Commun. Netw., 1, 721301.

Krawczyk, H., Canetti, R., \& Bellare, M. (1997). HMAC: Keyed-hashing for message authentication. IETF, Fremont, CA, USA, Tech. Rep. Rfc2104.

Krishna, P. V., Saritha, V., Vedha, G., Bhiwal, A., \& Chawla, A. S. (2012). Quality- of-service-enabled ant colonybased multipath routing for mobile ad hoc networks. IET Commun., 6(1), 76-83.

Marwaha, S., Indulska, J., \& Portmann M. (2009). Biologically inspired ant-based routing in mobile ad hoc networks (MANET): a survey symposia and workshops on ubiquitous, autonomic and trusted computing. QLD, Australia: Queensland Res. Lab. (QRL), Univ. of Queensland, Brisbane; p. 12-5, July.

Mehfuz, S., \& Doja, M. N. (2008). Swarm intelligent power-aware detection of unauthorized and compromised nodes in MANETs. J. Artif. Evolution Appl.

Misra, P., \& Enge, P. (2006). Global Positioning System: Signals, Measurements and Performance, 2nd ed. Lincoln, MA, USA: Ganga-Jamuna Press.

Misra, S., Dhurandher, S. K., Obaidat, M. S., Gupta, P., Verma, K., \& Narula, P. (2010). An ant swarm-inspired energy-aware routing protocol for wireless ad-hoc networks. J. Syst. Softw., 83(11), 2188-2199.

Neumann, F., \& Witt C. (2010). Ant colony optimization and the minimum spanning tree problem. Theoretical Computer Science, 411(25), 2406-2413.

Osagie, E., Thulasiraman, P., \& Thulasiram, R. K. (2008). PACONET: Improved ant colony optimization routing algorithm for mobile ad hoc networks. in Proc. 22nd Int. Conf. Adv. Inf. Netw. Appl. (AINA), pp. 204-211.

Panda, N. \& Panayak, B. K. (2018). Energy Aware Detection and Prevention of Black Hole Attack in MANET. International Journal of Engineering \& Technology (IJET), 7(2.6), 135-140.

Park, I., Jinguk, K., \& Ida, Pu. (2006). "Blocking expanding ring search algorithm for efficient energy consumption in mobile ad hoc networks." WONS 2006: Third annual conference on wireless on-demand network systems and services.

Peram, T., Kalyan, V., \& Chilukuri, K. M. (2003). Fitness-distance-ratio based particle swarm optimization. Swarm Intelligence Symposium, 2003. SIS'03. Proceedings of the 2003 IEEE. IEEE.

Prabaharan, S. B., \& Ponnusamy, R. (2016). Secure and energy efficient MANET routing incorporating trust values using hybrid ACO. in Proc. Int. Conf. Comput. Commun. Informat. (ICCCI), 1-8.

Rana G., Ballav, B., \& Pattanayak, B. K. (2015). Performance Analysis of Routing Protocols in Mobile Ad Hoc Networks, Proceedings of the International Conference on Information Technology (ICIT), pp.65-70.

Rana, H., Parimala, T., \& Ruppa, K. T. (2013). MAZACORNET: Mobility aware zone based ant colony optimization routing for VANET." 2013 IEEE Congress on Evolutionary Computation. IEEE.

Rangaraj, J., \& Anitha, M. (2017). Implementing Energy Optimization by a Novel Hybrid Technique for Performance Improvement in Mobile Ad Hoc Network. International Journal of Applied Engineering Research, 12.22 (2017), 12029-1203. 
Rath, M., Pati, B., \& Pattanayak, B. K. (2017). Cross Layer Based QoS Platform for Multimedia Transmission in MANET, Proceedings of 2017 11th International Conference on Intelligent Systems and Control, ISCO 2017, 506-509.

Sethi, S., \& Udgata, S. K. (2010). The efficient ant routing protocol for MANET. Int. J. Comput. Sci. Eng., 2(7), 2414-2420.

Sheibat, A., Seyed, A., Noudehi, A. N., \& Majdara, M. (2012). Solving Traveling Salesman Problem (TSP) using Ants Colony (ACO) Algorithm and comparing with Tabu Search, Simulated Annealing and Genetic Algorithm. Journal of Applied Sciences Research.

Vijayalakshmi, P., Francis, S. A. J., \& Dinakaran, J. A. (2015). A robust energy efficient ant colony optimization routing algorithm for multi-hop ad hoc networks in MANETs. Wireless Netw., 22(6), 1-20.

Vijayalakshmi, Sharmila, A. J. F., \& Abraham, D. J. (2014). "Max-Min-Path Energy-Efficient Routing AlgorithmA Novel Approach to Enhance Network Lifetime of MANETs." International Conference on Distributed Computing and Networking. Springer, Berlin, Heidelberg.

Vijayalakshmi, V., \& Palanivelu, T. (2007). Secure antnet routing algorithm for scalable adhoc networks using elliptic curve cryptography. J. Comput. Sci., 3(12), 939-943.

Wang, J., Osagie, E., Thulasiraman, P., \& Thulasiram, R. K. (2009). HOPNET: A hybrid ant colony optimization routing algorithm for mobile ad hoc network. Ad Hoc Netw., 7(4), 690-705.

Woungang, I., Obaidat, M. S., Dhurandher, S. K., Ferworn, A., \& Shah, W. (2013). An ant-swarm inspired energyefficient ad hoc on-demand routing protocol for mobile ad hoc networks. in Proc. IEEE Int. Conf. Commun. (ICC), pp. 3645-3649, Jul.

Zhou, J., Tan, H., Deng, Y., Cui, L., \& Liu, D. D. (2016). Ant colony-based energy control routing protocol for mobile ad hoc networks under different node mobility models. EURASIP J. Wireless Commun. Netw., 1, 105.

\section{Copyrights}

Copyright for this article is retained by the author(s), with first publication rights granted to the journal.

This is an open-access article distributed under the terms and conditions of the Creative Commons Attribution license (http://creativecommons.org/licenses/by/4.0/). 